SLAC-PUB-8210

July 1999

\title{
Nonlinear Longitudinal Waves in High Energy Stored Beams
}

\author{
Stephan I. Tzenov \\ Stanford Linear Accelerator Center, Stanford University, Stanford, CA 94309
}

\begin{abstract}
We solve the Vlasov equation for the longitudinal distribution function and find stationary wave patterns when the distribution in the energy error is Maxwellian. In the long wavelength limit a stability criterion for linear waves has been obtained and a Korteweg-de Vries- Burgers equation for the relevant hydrodynamic quantities has been derived.
\end{abstract}

Paper presented at Workshop on Instabilities of High Intensity Hadron Beams in Rings, Brookhaven National Laboratory

June 28 - July 1, 1999.

${ }^{*}$ Work supported by Department of Energy contract DE-AC03-76SF00515. 


\section{Introduction.}

Nonlinear wave interaction in high energy synchrotrons has recently received a great deal of attention (see e.g. [1], [2], [3]), since it has proven its importance for understanding a variety of phenomena in high intensity beams.

Perhaps, the simplest problem to study is the evolution in longitudinal direction only of a intense coasting beam influenced by a broad-band resonator type impedance. This model exhibits a surprisingly vast variety of interesting features, part of which have already been experimentally observed and theoretically investigated [1], [2], [3]. Different types of beam equilibria can be detected due to the collective (nonlinear) interaction between beam particles and resonator waves, the latter being induced by the beam itself. Solutions describing similar types of plasma equilibria [Bernstein-Greene-Kruskal (BGK) modes] are well-known in plasma physics [4]. Structures of arbitrary shape can be formed in the nonlinear stationary regime, which substantially depend on the type of the initial velocity distribution.

It is the purpose of the present paper to apply techniques borrowed from plasma physics to study nonlinear patterns in coasting beams that are in close analogy with BGK modes. In sections 3 and 4 we solve the Vlasov equation by expanding the distribution function in a power series of the resonator potential [5], and in the case of initial Maxwellian energy error distribution we obtain an equation, describing the evolution of stationary waves on the resonator. In section 5 we find a stability criterion for linear waves in the long wavelength limit and derive a Korteweg-de Vries-Burgers equation for the beam density, current velocity and resonator voltage.

\section{Model.}

We consider the longitudinal dynamics of a high energy stored beam governed by the set of equations [2], [3]:

$$
\begin{gathered}
\frac{\partial f}{\partial T}+v \frac{\partial f}{\partial \theta}+\lambda V \frac{\partial f}{\partial v}=0, \\
\frac{\partial^{2} V}{\partial T^{2}}+2 \gamma \frac{\partial V}{\partial T}+\omega^{2} V=\frac{\partial I}{\partial T}, \\
I(\theta ; T)=\int d v v f(\theta, v ; T) .
\end{gathered}
$$

The first equation (2.1) is the Vlasov equation for the longitudinal distribution function $f(\theta, v ; T)$ of an unbunched beam, while the second equation (2.2) governs the variation per turn of the voltage $V(\theta ; T)$ on a resonator. All dependent and independent variables, as well as free parameters in equations (2.1-3) are dimensionless and have been rescaled according to the relations:

$$
T=\omega_{s} t \quad ; \quad v=\frac{1}{\omega_{s}} \frac{d \theta}{d t}=1+\frac{k_{0} \Delta E}{\omega_{s}} \quad ; \quad \omega=\frac{\omega_{R}}{\omega_{s}},
$$




$$
\gamma=\frac{\omega}{2 Q} \quad ; \quad \lambda=\frac{e^{2} \mathcal{R} \gamma k_{0} \rho_{0}}{\pi} .
$$

Here $\omega_{s}$ is the angular revolution frequency of the synchronous particle, $\Delta E$ is the energy error, $\omega_{R}$ is the resonant frequency, $Q$ is the quality factor of the resonator, $\mathcal{R}$ is the resonator shunt impedance and $\rho_{0}$ is the uniform beam density distribution in the thermodynamic limit. Furthermore

$$
k_{0}=-\frac{\eta \omega_{s}}{\beta_{s}^{2} E_{s}}
$$

is the proportionality constant between the frequency deviation of a non synchronous particle with respect to the synchronous one, while $\eta=\alpha_{M}-\gamma_{s}^{-2}\left(\alpha_{M}-\right.$ momentum compaction factor) is the phase slip coefficient. The voltage variation per turn $V(\theta ; T)$, the beam current $I(\theta ; T)$ and the longitudinal distribution function $f(\theta, v ; T)$ entering equations (2.1-3) have been rescaled as well from their actual values $V_{a}(\theta ; T), I_{a}(\theta ; T)$ and $f_{a}(\theta, v ; T)$ as follows:

$$
V_{a}=2 e \omega_{s} \rho_{0} \gamma \mathcal{R} V \quad ; \quad I_{a}=e \omega_{s} \rho_{0} I \quad ; \quad f_{a}=\rho_{0} f .
$$

From the Vlasov equation (2.1) it is straightforward to obtain the continuity equation:

$$
\frac{\partial}{\partial T} \int d v f+\frac{\partial}{\partial \theta} \int d v v f=0
$$

which will be needed for the exposition in the next section.

\section{Solution of the Vlasov Equation.}

Let us now try to solve the Vlasov equation by the simple separation of variables ansatz:

$$
f(\theta, v ; T)=g(v) \psi(\theta ; T) .
$$

Substitution of (3.1) into the continuity equation (2.7) yields:

$$
\frac{\partial \psi}{\partial T}+\Omega \frac{\partial \psi}{\partial \theta}=0
$$

where

$$
\Omega=\frac{\int d v v g(v)}{\int d v g(v)} .
$$

The Vlasov equation (2.1) with (3.1-3) in hand can be further transformed to

$$
\frac{\partial \psi}{\partial \theta}=\frac{\lambda V \psi}{g(\Omega-v)} \frac{d g}{d v} .
$$

The separation of variables ansatz (3.1) implies 


$$
\frac{d g}{d v}=\frac{\Omega-v}{\sigma_{v}^{2}} g
$$

which leads to the well-known equilibrium Maxwell-Boltzmann distribution:

$$
\begin{gathered}
g(v)=\frac{1}{\sigma_{v} \sqrt{2 \pi}} \exp \left[-\frac{(v-\Omega)^{2}}{2 \sigma_{v}^{2}}\right], \\
\psi(\theta ; T)=\mathcal{Z} \exp \left[\frac{\lambda \varphi(\theta ; T)}{\sigma_{v}^{2}}\right] \quad ; \quad V(\theta ; T)=\frac{\partial \varphi(\theta ; T)}{\partial \theta},
\end{gathered}
$$

where

$$
\mathcal{Z}^{-1}=\int_{0}^{2 \pi} d \theta \exp \left[\frac{\lambda \varphi(\theta ; T)}{\sigma_{v}^{2}}\right] .
$$

The solution (3.4-6) suggests further generalization [5] of the separation of variables ansatz (3.1)

$$
f(\theta, v ; T)=\sum_{k=0}^{\infty} g_{k}(v) \varphi^{k}(\theta ; T) .
$$

Instead of equations (3.2) and (3.3) we now have

$$
\frac{\partial \varphi}{\partial T}+\Omega(\theta ; T) \frac{\partial \varphi}{\partial \theta}=0
$$

where

$$
\begin{gathered}
\Omega(\theta ; T)=\frac{\sum_{k=1}^{\infty} k \mathcal{A}_{k} \varphi^{k-1}(\theta ; T)}{\sum_{k=1}^{\infty} k \mathcal{B}_{k} \varphi^{k-1}(\theta ; T)} . \\
\mathcal{A}_{k}=\int \operatorname{dvvg}_{k}(v) \quad ; \quad \mathcal{B}_{k}=\int d v g_{k}(v) .
\end{gathered}
$$

In order to determine the yet unknown functions $g_{k}(v)$ we make the assumption:

$$
\Omega(\theta ; T)=\text { const }
$$

which will be proved a posteriori to hold and substitute (3.7) into the Vlasov equation (2.1). Taking into account (3.2a) we obtain:

$$
(v-\Omega) \sum_{k=1}^{\infty} k g_{k}(v) \varphi^{k-1}(\theta ; T)+\lambda \sum_{k=0}^{\infty} \frac{d g_{k}(v)}{d v} \varphi^{k}(\theta ; T)=0 .
$$

Equating coefficients in front of powers of $\varphi$ yields the following recurrence relation

$$
(v-\Omega)(k+1) g_{k+1}(v)=-\lambda \frac{d g_{k}(v)}{d v},
$$


or

$$
g_{k+1}(v)=\frac{\lambda}{k+1} \widehat{\mathcal{D}} g_{k}(v),
$$

where we have introduced the operator [5]

$$
\widehat{\mathcal{D}}=\frac{1}{\Omega-v} \frac{d}{d v}
$$

Noting that the formal solution of the recurrence relation (3.11) has the form

$$
g_{k}(v)=\frac{\lambda^{k}}{k !} \widehat{\mathcal{D}}^{k} g_{0}(v)
$$

we finally arrive at the general solution of the Vlasov equation

$$
f(\theta, v ; T)=\sum_{k=0}^{\infty} \frac{\lambda^{k} \varphi^{k}(\theta ; T)}{k !} \widehat{\mathcal{D}}^{k} g_{0}(v) .
$$

What remains now is to verify the condition (3.9). It suffices to note that [5]

$$
\begin{gathered}
\mathcal{A}_{k}=\frac{\lambda^{k}}{k !} \int d v v \widehat{\mathcal{D}}^{k} g_{0}(v)=\frac{\lambda^{k}}{k !} \int d v \frac{v}{\Omega-v} \frac{d}{d v}\left[\widehat{\mathcal{D}}^{k-1} g_{0}(v)\right]= \\
=-\frac{\lambda^{k} \Omega}{k !} \int \frac{d v}{(\Omega-v)^{2}} \widehat{\mathcal{D}}^{k-1} g_{0}(v)
\end{gathered}
$$

and similarly

$$
\mathcal{B}_{k}=-\frac{\lambda^{k}}{k !} \int \frac{d v}{(\Omega-v)^{2}} \widehat{\mathcal{D}}^{k-1} g_{0}(v)
$$

Thus

$$
\mathcal{A}_{k}=\Omega \mathcal{B}_{k}
$$

which proves equation (3.9).

Clearly the solution (3.14) is uniquely determined by the generic function $g_{0}(v)$. The simplest choice is when $g_{0}(v)$ is the Maxwellian (3.4), that is $g_{0}(v)$ itself is an eigenfunction of the operator $\widehat{\mathcal{D}}$ with an eigenvalue $\sigma_{v}^{-2}$ [c.f. equation (3.4a)]. In this case we immediately recover the distribution (3.1) with (3.4-6).

\section{Nonlinear Stationary Waves.}

In order to derive an equation for the potential $\varphi(\theta ; T)$ we insert (3.1) and (3.4-6) into (2.2) and obtain:

$$
\frac{\partial^{3} \varphi}{\partial \theta \partial T^{2}}+2 \gamma \frac{\partial^{2} \varphi}{\partial \theta \partial T}+\omega^{2} \frac{\partial \varphi}{\partial \theta}=\mathcal{Z} \Omega \frac{\partial}{\partial T}\left[\exp \left(\frac{\lambda \varphi}{\sigma_{v}^{2}}\right)\right]
$$


Making use of relation (3.2a) we cast equation (4.1) into the form

$$
\frac{\partial^{3} \varphi}{\partial T^{3}}+2 \gamma \frac{\partial^{2} \varphi}{\partial T^{2}}+\omega^{2} \frac{\partial \varphi}{\partial T}=-\mathcal{Z} \Omega^{2} \frac{\partial}{\partial T}\left[\exp \left(\frac{\lambda \varphi}{\sigma_{v}^{2}}\right)\right] .
$$

Integrating once equation (4.2) with due account of the initial condition

$$
\varphi(\theta ; T=0)=\frac{\partial \varphi(\theta ; T=0)}{\partial T}=\frac{\partial^{2} \varphi(\theta ; T=0)}{\partial T^{2}}=0
$$

we obtain

$$
\frac{\partial^{2} \varphi}{\partial T^{2}}+2 \gamma \frac{\partial \varphi}{\partial T}+\omega^{2} \varphi=\mathcal{Z} \Omega^{2}\left[1-\exp \left(\frac{\lambda \varphi}{\sigma_{v}^{2}}\right)\right] .
$$

Expanding the factor in square brackets on the right-hand-side of equation (4.4) around the stationary solution $\varphi_{s}=0$ yields

$$
\frac{\partial^{2} \varphi}{\partial T^{2}}+2 \gamma \frac{\partial \varphi}{\partial T}+\omega^{2} \varphi=-\frac{\lambda \mathcal{Z} \Omega^{2}}{\sigma_{v}^{2}} \varphi\left(1+\frac{\lambda \varphi}{2 \sigma_{v}^{2}}+\frac{\lambda^{2} \varphi^{2}}{6 \sigma_{v}^{4}}+\ldots\right) .
$$

Above the transition energy $\gamma_{s}>\gamma_{T}\left(\gamma_{T}=\alpha_{M}^{-1 / 2}\right)$ the parameter $\lambda$ is negative, so that two cases can be distinguished. Defining

$$
\omega_{0}=\omega^{2}-\frac{|\lambda| \mathcal{Z} \Omega^{2}}{\sigma_{v}^{2}}
$$

we can state the two cases mentioned above in a more explicit way:

Case I: Provided $\omega_{0}>0$, equation (4.5) can be transformed to a damped Duffing equation with an additional quadratic nonlinearity

$$
\frac{\partial^{2} \varphi}{\partial T^{2}}+2 \gamma \frac{\partial \varphi}{\partial T}+\left|\omega_{0}\right| \varphi=-\frac{\lambda^{2} \mathcal{Z} \Omega^{2}}{2 \sigma_{v}^{4}}\left(\varphi^{2}-\frac{|\lambda|}{3 \sigma_{v}^{2}} \varphi^{3}\right) .
$$

Case II: For $\omega_{0}<0$ equation (4.5) takes the form

$$
\frac{\partial^{2} \varphi}{\partial T^{2}}+2 \gamma \frac{\partial \varphi}{\partial T}-\left|\omega_{0}\right| \varphi=-\frac{\lambda^{2} \mathcal{Z} \Omega^{2}}{2 \sigma_{v}^{4}}\left(\varphi^{2}-\frac{|\lambda|}{3 \sigma_{v}^{2}} \varphi^{3}\right) .
$$

In the limit $\gamma \rightarrow 0$ equation (4.8) can be solved when neglecting the cubic term. The result is:

$$
\varphi(\theta ; T)=\frac{3\left|\omega_{0}\right| \sigma_{v}^{4}}{\lambda^{2} \mathcal{Z} \Omega^{2} \cosh ^{2}\left[\frac{\sqrt{\left|\omega_{0}\right|}}{2 \Omega}(\theta-\Omega T)\right]} .
$$

This is a drifting hump-like structure that is well-known as a solitary wave of the Korteweg-de Vries (KdV) type. 


\section{The Korteweg-de Vries-Burgers Equation.}

The exact solution of the Vlasov equation obtained in the preceding sections was found based on the stationary wave condition given by the continuity equation (3.2). In order to provide a more general treatment of the problem we introduce the new coordinates and variables along with the moving beam particles

$$
z=\theta-T \quad ; \quad u=v-1 .
$$

Then the basic equations (2.1-3) can be written as:

$$
\begin{gathered}
\frac{\partial f}{\partial \theta}+u \frac{\partial f}{\partial z}+\lambda V \frac{\partial f}{\partial u}=0 \\
\frac{\partial^{2} V}{\partial z^{2}}-2 \gamma \frac{\partial V}{\partial z}+\omega^{2} V=-\frac{\partial}{\partial z} \int d u(1+u) f(z, u ; \theta) .
\end{gathered}
$$

Let us now pass to the hydrodynamic description of the longitudinal beam motion. The gas dynamic equations read as

$$
\begin{gathered}
\frac{\partial F}{\partial \theta}+\frac{\partial}{\partial z}(F U)=0 \\
\frac{\partial U}{\partial \theta}+U \frac{\partial U}{\partial z}=\lambda V-\frac{\sigma_{v}^{2}}{F} \frac{\partial F}{\partial z} \\
\frac{\partial^{2} V}{\partial z^{2}}-2 \gamma \frac{\partial V}{\partial z}+\omega^{2} V=\frac{\partial F}{\partial \theta}-\frac{\partial F}{\partial z}
\end{gathered}
$$

where

$$
F(z ; \theta)=\int d u f(z, u ; \theta) \quad ; \quad F(z ; \theta) U(z ; \theta)=\int d u u f(z, u ; \theta) .
$$

Obviously the stationary solution of the gas dynamic equations (5.4-6) is given by

$$
F_{0}=1 \quad ; \quad U_{0}=0 \quad ; \quad V_{0}=0 .
$$

The dispersion law of linear waves of the form

$$
(F, U, V)=\left(F_{L}, U_{L}, V_{L}\right) \exp [i(\Omega \theta-k z)]
$$

is governed by the following equation

$$
1-i \lambda Z(k) \frac{k+\Omega}{\Omega^{2}-k^{2} \sigma_{v}^{2}}=0
$$

where $Z(k)$ is the well-known impedance function

$$
Z(k)=\frac{i k}{k^{2}+2 i \gamma k-\omega^{2}} .
$$


In the long wavelength limit (small $k$ ) the dispersion equation (5.8) has two roots given by the expression

$$
\Omega_{1,2}=\frac{k}{2 \omega^{2}}\left(\lambda \pm \sqrt{\lambda^{2}+4 \lambda \omega^{2}+4 \omega^{4} \sigma_{v}^{2}}\right)
$$

which are real below transition energy. However, the situation when the energy of the synchronous particle is above transition energy is different. The solutions (5.10) to the dispersion equation are real, provided

$$
|\lambda| \leq \lambda_{1} \quad ; \quad|\lambda| \geq \lambda_{2} \quad ; \quad \lambda_{1,2}=2 \omega^{2}\left(1 \mp \sqrt{1-\sigma_{v}^{2}}\right) .
$$

An instability occurs when $\Omega_{1,2}$ are complex, that is when

$$
\lambda_{1}<|\lambda|<\lambda_{2}
$$

In what follows we will study the case when our system is linearly stable, that is either below transition energy or in the stability region (5.11).

The solution of the dispersion equation in the long wavelength limit suggests that new scaled coordinates should be introduced [6], [7]

$$
\sigma=\sqrt{\epsilon}(z-\alpha \theta) \quad ; \quad \chi=\epsilon^{3 / 2} \theta,
$$

where $\epsilon$ is a formal small parameter. Then the gas dynamic equations can be rewritten as

$$
\begin{gathered}
-\alpha \frac{\partial F}{\partial \sigma}+\frac{\partial}{\partial \sigma}(F U)+\epsilon \frac{\partial F}{\partial \chi}=0 \\
-\alpha \frac{\partial U}{\partial \sigma}+U \frac{\partial U}{\partial \sigma}+\epsilon \frac{\partial U}{\partial \chi}=\lambda \widetilde{V}-\frac{\sigma_{v}^{2}}{F} \frac{\partial F}{\partial \sigma}, \\
\epsilon \frac{\partial^{2} \widetilde{V}}{\partial \sigma^{2}}-2 \epsilon \gamma_{0} \frac{\partial \widetilde{V}}{\partial \sigma}+\omega^{2} \widetilde{V}=-(1+\alpha) \frac{\partial F}{\partial \sigma}+\epsilon \frac{\partial F}{\partial \chi}
\end{gathered}
$$

where

$$
\begin{gathered}
V=\sqrt{\epsilon} \tilde{V} \quad ; \quad \gamma=\sqrt{\epsilon} \gamma_{0}, \\
\omega^{2} \alpha^{2}-\lambda \alpha-\lambda-\omega^{2} \sigma_{v}^{2}=0 \quad ; \quad\left(\alpha=\frac{\Omega_{1,2}}{k}\right) .
\end{gathered}
$$

Assuming the perturbation expansions:

$$
F=1+\sum_{m=1}^{\infty} \epsilon^{m} F_{m} \quad ; \quad U=\sum_{m=1}^{\infty} \epsilon^{m} U_{m} \quad ; \quad \tilde{V}=\sum_{m=1}^{\infty} \epsilon^{m} V_{m}
$$

for the first and second-order terms in $\epsilon$ we obtain respectively 


$$
\frac{\partial U_{1}}{\partial \sigma}=\alpha \frac{\partial F_{1}}{\partial \sigma}=\frac{\alpha \lambda V_{1}}{\sigma_{v}^{2}-\alpha^{2}}
$$

or

$$
U_{1}(\sigma, \chi)=\alpha F_{1}(\sigma, \chi)+G(\chi),
$$

where $G(\chi)$ is a generic function of the variable $\chi$, and

$$
\begin{gathered}
-\alpha \frac{\partial F_{2}}{\partial \sigma}+\frac{\partial U_{2}}{\partial \sigma}+\frac{\partial}{\partial \sigma}\left(F_{1} U_{1}\right)+\frac{\partial F_{1}}{\partial \chi}=0 \\
-\alpha \frac{\partial U_{2}}{\partial \sigma}+U_{1} \frac{\partial U_{1}}{\partial \sigma}+\frac{\partial U_{1}}{\partial \chi}=\lambda V_{2}-\sigma_{v}^{2} \frac{\partial F_{2}}{\partial \sigma}+\sigma_{v}^{2} F_{1} \frac{\partial F_{1}}{\partial \sigma} \\
\frac{\partial^{2} V_{1}}{\partial \sigma^{2}}-2 \gamma_{0} \frac{\partial V_{1}}{\partial \sigma}+\omega^{2} V_{2}=-(1+\alpha) \frac{\partial F_{2}}{\partial \sigma}+\frac{\partial F_{1}}{\partial \chi}
\end{gathered}
$$

Eliminating $F_{2}, U_{2}$ and $V_{2}$ from equations (5.22) we finally arrive at the Korteweg-de VriesBurgers equation

$$
\frac{\partial F_{1}}{\partial \chi}+\left(c_{1} F_{1}+c_{2} G\right) \frac{\partial F_{1}}{\partial \sigma}+D \frac{\partial^{3} F_{1}}{\partial \sigma^{3}}-2 \gamma D \frac{\partial^{2} F_{1}}{\partial \sigma^{2}}=h \frac{d G}{d \chi}
$$

where

$$
\begin{array}{cc}
c_{1}=\frac{\omega^{2}\left(3 \alpha^{2}-\sigma_{v}^{2}\right)}{2 \alpha \omega^{2}-\lambda} \quad ; \quad & c_{2}=\frac{2 \alpha \omega^{2}}{2 \alpha \omega^{2}-\lambda}, \\
D=\frac{\sigma_{v}^{2}-\alpha^{2}}{2 \alpha \omega^{2}-\lambda} \quad ; \quad h=\frac{\omega^{2}}{\lambda-2 \alpha \omega^{2}} .
\end{array}
$$

It is important to note that $\alpha^{-1} U_{1}$ and $\lambda\left(\sigma_{v}^{2}-\alpha^{2}\right)^{-1} \int d \sigma V_{1}$ satisfy exactly the same equation (5.23).

Similar Korteweg-de Vries-Burgers equation in the case below transition energy has been recently derived by A. Aceves employing the method of multiple scales [8].

\section{Concluding Remarks.}

We have studied the longitudinal dynamics of a high energy coasting beam moving in a resonator. The coupled Vlasov equation for the longitudinal distribution function and the equation for the resonator voltage have been solved by closely following the method of Karimov and Lewis [5]. The key point of this method consists in the representation of the distribution function as a power series in the resonator potential. Further self-consistent stationary wave patterns have been found in the simplest equilibrium case of Maxwellian distribution in the energy error. 
In the long wavelength (small wavenumber) limit a stability criterion for linear waves has been obtained and a Korteweg-de Vries-Burgers equation for the relevant hydrodynamic quantities has been derived.

An important (and interesting) extension of the results obtained here involves the longitudinal dynamics of a bunched beam. These will be reported elsewhere.

\section{Acknowledgements.}

I would like to thank A. Aceves and P. Colestock for many helpful discussions concerning the subject of the present paper.

This work was supported by the US Department of Energy, Office of Basic Energy Sciences, under contract DE-AC03-76SF00515.

\section{References}

[1] P.L. Colestock, L.K. Spentzouris and S.I. Tzenov, "Coherent Nonlinear Phenomena in High Energy Synchrotrons: Observations and Theoretical Models.", In International Symposium on Near Beam Physics, R.A. Carrigan and N.V. Mokhov eds., Fermilab, June 1998, pp 94-104.

[2] S.I. Tzenov and P.L. Colestock, "Solitary Waves on a Coasting High-Energy Stored Beam.", FERMILAB-Pub-98/258, Fermilab, September 1998.

[3] S.I. Tzenov, "Formation of Patterns and Coherent Structures in Charged Particle Beams.", FERMILAB-Pub-98/275, Fermilab, October 1998.

[4] I.B. Bernstein, J.M. Greene and M.D. Kruskal, Phys. Rev. 108, (1957) p. 546.

[5] A.R. Karimov and H.R. Lewis, Phys. Plasmas, 6, (1999) p. 759.

[6] H. Washimi and T. Taniuti, Phys. Rev. Lett., 17, (1966) p. 996

[7] Lokenath Debnath, "Nonlinear Partial Differential Equations for Scientists and Engineers.", Birkhauser, Boston, 1997.

[8] A. Aceves, To be published. 